\title{
Management of an acute catecholamine- induced cardiomyopathy and circulatory collapse: a multidisciplinary approach
}

\author{
R T Casey', B G Challis', D Pitfield1, R M Mahroof2, N Jamieson³, C J Bhagra4, \\ A Vuylsteke $^{5}$, S J Pettit6 and K C Chatterjee ${ }^{1}$
}

Departments of ${ }^{1}$ Endocrinology and Diabetes, ${ }^{2}$ Anaesthetics, ${ }^{3} \mathrm{Hepatobiliary}$ and Pancreatic Surgery, ${ }^{4} \mathrm{Cardiology}$, Cambridge University NHS Foundation Trust, Cambridge, UK, ${ }^{5} \mathrm{Critical}$ Care Unit, and ${ }^{6} \mathrm{Advanced}$ Heart Failure Unit, Papworth Hospital NHS Foundation Trust, Cambridge, UK

Correspondence should be addressed to R T Casey

Email

ruthcasey232@gmail.com

\section{Summary}

A phaeochromocytoma (PC) is a rare, catecholamine-secreting neuroendocrine tumour arising from the adrenal medulla. Presenting symptoms of this rare tumour are highly variable but life-threatening multiorgan dysfunction can occur secondary to catecholamine-induced hypertension or hypotension and subsequent cardiovascular collapse. High levels of circulating catecholamines can induce an acute stress cardiomyopathy, also known as Takotsubo cardiomyopathy. Recent studies have focused on early diagnosis and estimation of the prevalence of acute stress cardiomyopathy in patients with PC, but very little is reported about management of these complex cases. Here, we report the case of a 38-year-old lady who presented with an acute Takotsubo or stress cardiomyopathy and catecholamine crisis, caused by an occult left-sided $5 \mathrm{~cm}$ PC. The initial presenting crisis manifested with symptoms of severe headache and abdominal pain, triggered by a respiratory tract infection. On admission to hospital, the patient rapidly deteriorated, developing respiratory failure, cardiogenic shock and subsequent cardiovascular collapse due to further exacerbation of the catecholamine crisis caused by a combination of opiates and intravenous corticosteroid. An echocardiogram revealed left ventricular apical hypokinesia and ballooning, with an estimated left ventricular ejection fraction of $10-15 \%$. Herein, we outline the early stabilisation period, preoperative optimisation and intraoperative management, providing anecdotal guidance for the management of this rare life-threatening complication of PC.

\section{Learning points:}

- A diagnosis of phaeochromocytoma should be considered in patients presenting with acute cardiomyopathy or cardiogenic shock without a clear ischaemic or valvular aetiology.

- Catecholamine crisis is a life-threatening medical emergency that requires cross-disciplinary expertise and management to ensure the best clinical outcome.

- After initial resuscitation, treatment of acute catecholamine-induced stress cardiomyopathy requires careful introduction of alpha-blockade followed by beta-blockade if necessary to manage $\beta$-receptor-mediated tachycardia.

- Prolonged $\alpha$-adrenergic receptor stimulation by high levels of circulating catecholamines precipitates arterial vasoconstriction and intravascular volume contraction, which can further exacerbate hypotension. Invasive pressure monitoring can aid management of intravascular volume in these complex patients. 


\section{Background}

Phaeochromocytoma (PC) typically presents with symptoms of catecholamine excess including headache, palpitations, paroxysmal hypertension, anxiety and excessive sweating. However, the severity of these symptoms is variable, owing, in part, to the heterogenous genetic background of these tumours, and increasingly PC tumours are diagnosed incidentally following crosssectional imaging (1). A catecholamine crisis is defined as acute and severe haemodynamic instability and collapse (2) and is a rare, but dreaded, complication of PC. A recent multicentre retrospective review by Resiter et al. reported that $11 \%$ of the patients with PC presented with a catecholamine crisis (3) and a recent review reported a pooled mortality rate of $15 \%$ (4). Acute stress cardiomyopathy is one of the most commonly reported complications of a catecholamine crisis $(3,4,5)$ and is defined as an acute-reversible regional ventricular wall motion abnormality with a characteristic circular pattern, incongruent with the coronary artery supply distribution $(6,7)$.

Convergent evidence over the past fifteen years suggests that this distinct form of cardiomyopathy is caused by hyperstimulation of the cardiac sympathetic nerve terminals, due to physiological production of catecholamines during severe stress or autonomous pathological secretion from a PC (6). Chest pain is the most common presenting feature of acute stress cardiomyopathy and patients with an underlying PC may report symptoms related to excess catecholamine secretion (8). Acute stress cardiomyopathy due to PC typically presents at a younger age, is more commonly associated with an apical regional wall motion abnormality (8) and is associated with a lower mean left ventricular ejection fraction compared with acute stress cardiomyopathy due to other causes (8), as evident in this case. Early cautious treatment with alpha-blockade remains the mainstay of treatment for a catecholamine crisis including acute stress cardiomyopathy (4), with the aim of reducing peripheral vasoconstriction and preventing arrhythmia provocation. Indeed, a review of the published literature has revealed a correlation between alpha-blockade administration and improved survival in patients with catecholamine crises (4). However, as refractory hypotension is a common feature of a catecholamine crisis and acute stress cardiomyopathy, early fluid resuscitation, invasive pressure monitoring and cross-speciality input from cardiology, endocrinology and intensive care are crucial to enable sustained treatment with alpha-blockade therapy.

\section{Case presentation}

We present the case of a 37-year-old lady originally from China, who presented with a one-day history of epigastric pain, vomiting and severe headache. On initial assessment in the emergency department, she was noted to be pale and clammy with a heart rate of 80 beats per minute (bpm) and a blood pressure of $145 / 90 \mathrm{mmHg}$. She had no past medical history and was not taking any prescribed medications, over the counter or herbal medications. There was no significant family history. She lived with her husband and two children; of note, her youngest child was ten months old and the patient reported a similar headache following normal vaginal delivery with this child, but there was no history of hypertension or other cardiovascular symptoms in this pregnancy.

On examination, crepitations were auscultated in both lung bases and she had epigastric tenderness with no guarding and audible bowel sounds. She was noted to be euvolemic and haemodynamically stable and as she had clinical findings in both lungs and abdomen, a computed tomography (CT) examination of chest and abdomen was undertaken. On return from the CT scan, the patient had deteriorated significantly and had become tachypnoeic (respiratory rate of 35 ) with a sinus tachycardia (135 bpm). The CT scan demonstrated pseudonodular opacities with ground glass changes in both lungs (Fig. 1A) and a $5-\mathrm{cm}$ left-sided adrenal lesion (Fig. 1B). The patient was initially treated for an atypical pneumonia with intravenous antibiotics and non-invasive ventilation using bi-level positive airway pressure (BIPAP). After transfer to a high dependency unit, a bedside transthoracic echocardiogram was performed, which revealed hypokinetic mid-inferior and apical segments and severely reduced left ventricular ejection fraction estimated at 10-15\%. No valvular abnormality was detected (Fig. 2A and B).

The differential diagnoses included acute myocarditis and the patient was treated with intravenous methylprednisolone (1 g) which was abruptly followed by further severe clinical deterioration with an increase in her respiratory rate $(45 \mathrm{bpm})$ and a drop in blood pressure (106/60 mmHg). Arterial blood gas analysis revealed a lactic acidosis and a markedly elevated troponin (Table 1). An electrocardiogram demonstrated lateral and inferior non-specific $t$-wave changes in addition to a sinus tachycardia and a prolonged QT interval (Fig. 1C). The patient was transferred to a specialist cardiac intensive care unit in anticipation that mechanical circulatory support may be required. 


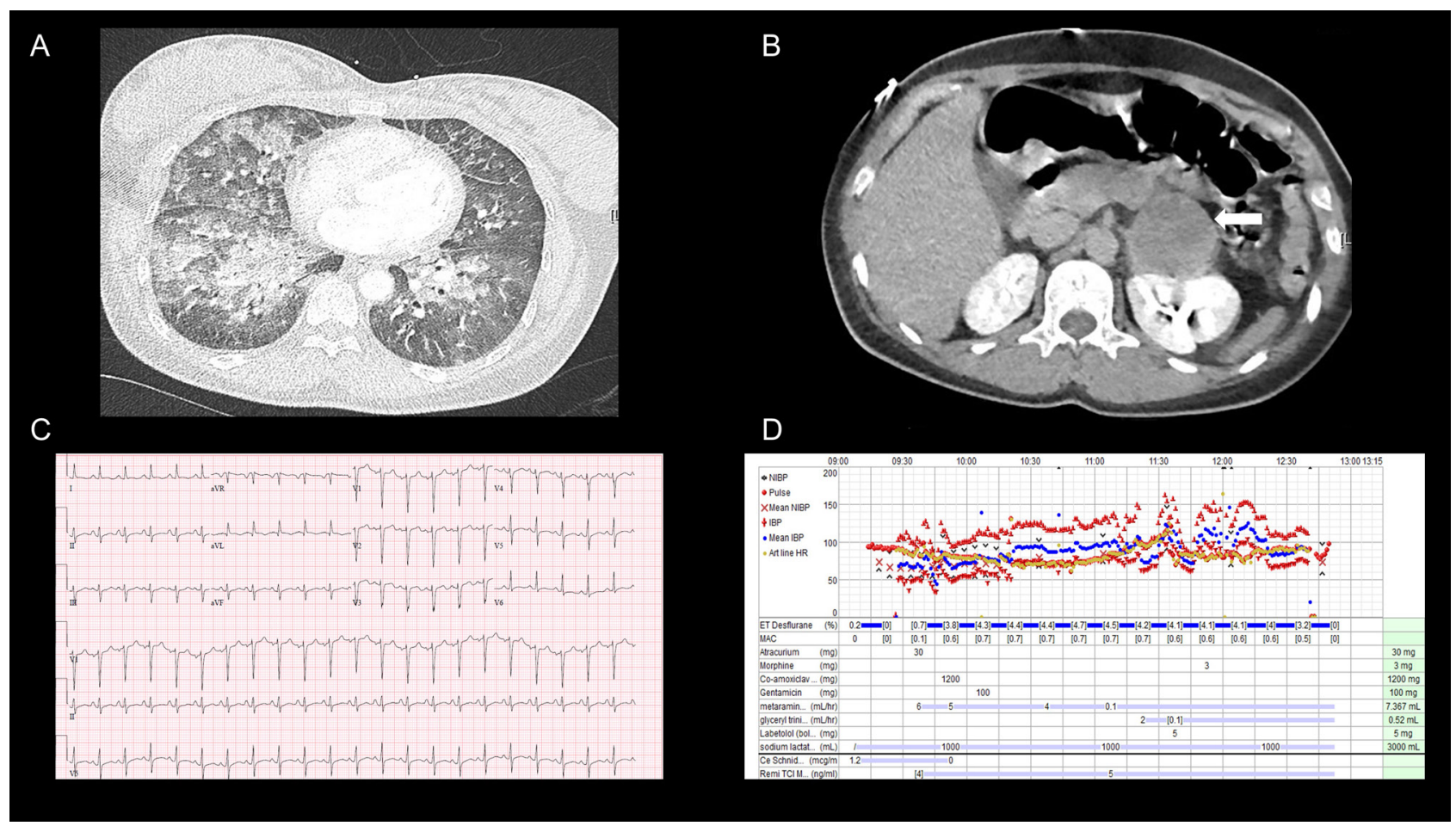

Figure 1

(A) A coronal section of the contrast-enhanced CT thorax performed on day 1 of admission and demonstrates bilateral pseudonodular opacities with ground glass changes, in keeping with pulmonary oedema, (B) A coronal section of the abdomen on the same CT exam and the 5-cm left-sided adrenal mass is highlighted by the white arrow. (C) The electrocardiogram demonstrating non-specific $t$-wave changes, a prolonged QT interval and a sinus tachycardia. (D) The intraoperative monitoring and the additional medications administered over the period of surgery.

Following transfer, an endocrine opinion was subsequently sought regarding the left adrenal mass. Subsequent plasma and urinary metanephrines revealed a marked elevation in both metanephrine and normetanephrine levels (Table 1), thereby supporting the diagnosis of a left-sided PC and a catecholamine crisis.

Cardiac function improved within a 24-h period, following the introduction of alpha-blockade (phenoxybenzamine up-titrated to $20 \mathrm{mg}$ daily). The regional wall motion abnormality, initially detected by echocardiogram, had resolved with an improvement in the ejection fraction to $45-50 \%$ prior to surgery, fourteen days after the initial presentation. Cardiac MRI showed improvement in LV systolic function with no myocardial oedema on T2-weighted sequences (Fig. 2B), and no late gadolinium enhancement to suggest myocardial fibrosis (Fig. 2C). The diagnosis of PC was confirmed following histological examination of the resected tumour following an uncomplicated left adrenalectomy, with evidence of a $5 \mathrm{~cm}$ haemorrhagic PC, with a low ki67\% proliferation index $(<1 \%)$ and preserved SDHB immunohistochemistry. Subsequent genetic analysis using next-generation sequencing did not reveal a mutation in a panel of ten genes analysed (SDHA, SDHB, SDHC, SDHD, SDHAF2, MAX, VHL, FH, RET, TEMEM127).

\section{Investigation}

\section{Treatment}

1. Initial treatment: Co-amoxiclav and clarithromycin were commenced for a presumed atypical pneumonia and a single dose of $1 \mathrm{~g}$ of methylprednisolone (IV) was given for a suspected diagnosis of acute myocarditis. Non-invasive ventilation with BIPAP with settings of 15 for inspiratory pulmonary airway pressure and 5 for expiratory was commenced following the initial clinical deterioration and continued for the first $48 \mathrm{~h}$. A furosemide infusion of $80 \mathrm{mg} / 24 \mathrm{~h}$ was initiated after the echocardiogram revealed a severely reduced ejection fraction in addition to a low dose $(0.3 \mathrm{mg} / \mathrm{h})$ nitrate infusion using glyceryl trinitrate (GTN). The later deterioration in haemodynamic stability prompted cessation of therapy with furosemide and GTN infusions. 


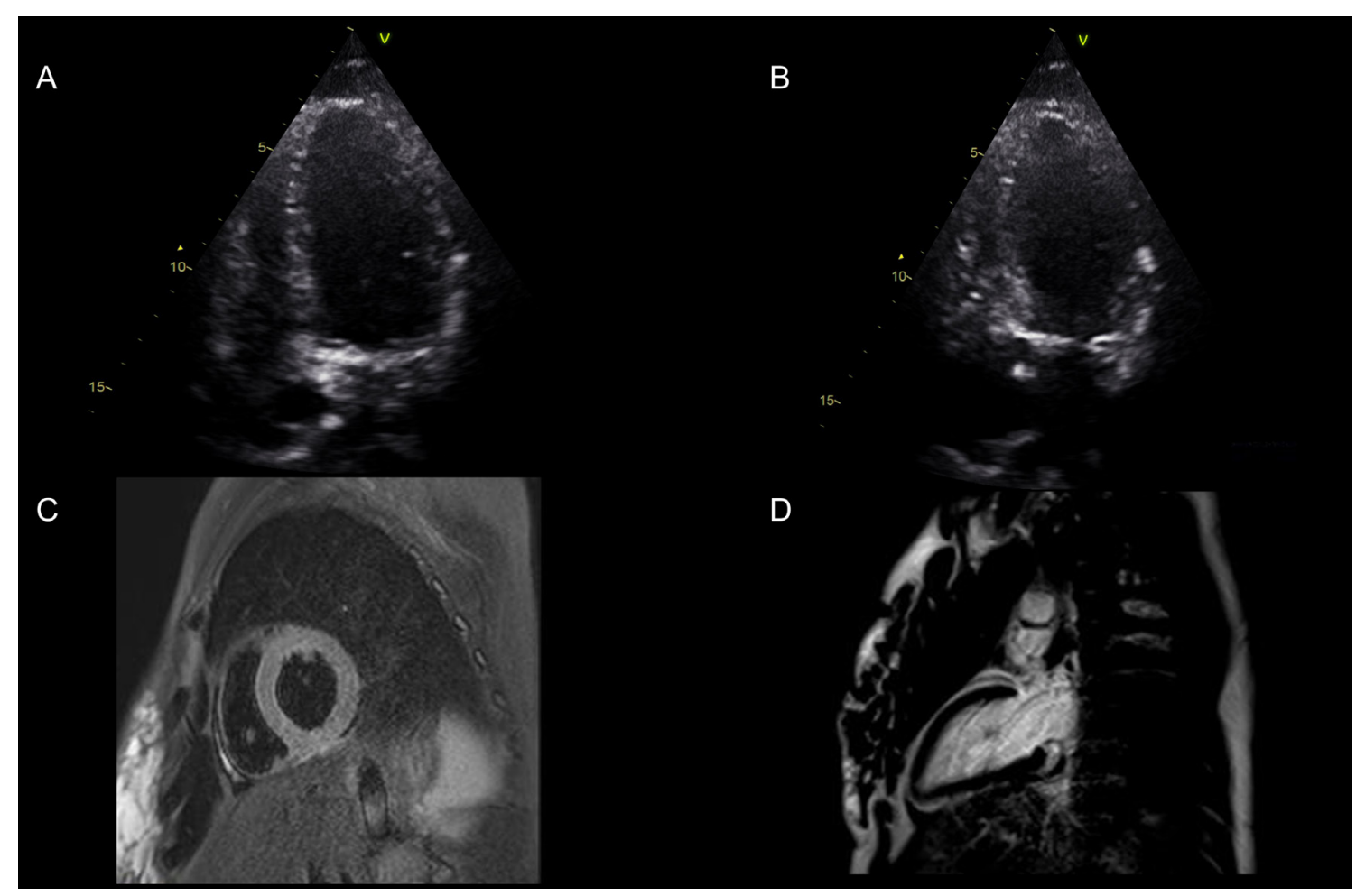

\section{Figure 2}

Images (A) and (B) display the apical 4 chamber view in end diastole and end systole The basal anteroseptal, basal inferolateral and basal inferior segments had reasonable contractility, and the mid-inferior segment was hypokinetic. The remaining segments were severely hypokinetic/akinetic. Visually estimated LVEF $=10-15 \%$. (C and D) Cardiac MRI showed improvement in LV systolic function with no myocardial oedema on T2-weighted sequences (Fig. 2C), and no late gadolinium enhancement to suggest myocardial fibrosis (Fig. 2D).

Table 1 Biochemical and hematological investigations performed.

\begin{tabular}{|c|c|c|}
\hline Investigation & Result & Reference range \\
\hline White cell count & 21.7 & $3.9-10.210 \times 9 / L$ \\
\hline Neutrophil count & 18.6 & $1.50-7.7010 \times 9 / L$ \\
\hline Haemoglobin & 143.5 & $115-178 \mathrm{~g} / \mathrm{L}$ \\
\hline Sodium & 139 & $135-145 \mathrm{mmol} / \mathrm{L}$ \\
\hline Potassium & 3.8 & $3.5-5.1 \mathrm{mmol} / \mathrm{L}$ \\
\hline Urea & 6.4 & $2.5-7.8 \mathrm{mmol} / \mathrm{L}$ \\
\hline Creatinine & 59 & $44-97 \mu \mathrm{mol} / \mathrm{L}$ \\
\hline Troponin & 8744 & $0-56 \mathrm{ng} / \mathrm{L}$ \\
\hline C-reactive protein & 11.1 & $0-6 \mathrm{mg} / \mathrm{L}$ \\
\hline Free thyroxine hormone & 14.1 & $10.0-19.8 \mathrm{pmol} / \mathrm{L}$ \\
\hline $\begin{array}{l}\text { Thyroid stimulating } \\
\text { hormone }\end{array}$ & 0.09 & $0.35-5.50 \mathrm{mU} / \mathrm{L}$ \\
\hline Creatinine Kinase & 441 & $25-200 \mathrm{U} / \mathrm{L}$ \\
\hline $\begin{array}{l}\text { Lactate on arterial blood } \\
\text { gas }\end{array}$ & 7.1 & $0.6-1.4 \mathrm{mmol} / \mathrm{L}$ \\
\hline $\mathrm{pH}$ on arterial blood gas & 7.20 & $7.35-7.45$ \\
\hline $\begin{array}{l}\text { Bicarbonate on arterial } \\
\text { blood gas }\end{array}$ & 18.7 & \\
\hline Plasma normetanephrine & 10523 & $<2500 \mathrm{pmol} / \mathrm{L}$ \\
\hline Plasma metanephrine & 3026 & $<900 \mathrm{pmol} / \mathrm{L}$ \\
\hline Urine metanephrine & 20815 & $0-2000 \mathrm{nmol} / 24 \mathrm{~h}$ \\
\hline Urine normetanephrine & 16905 & $0-4900 \mathrm{nmol} / 24 \mathrm{~h}$ \\
\hline
\end{tabular}

2. Preoperative treatment of PC: The patient was managed at a high dependency unit for the preoperative stabilisation and immediate post-operative period. Invasive monitoring with an arterial catheter, a central venous catheter and a pulmonary artery flotation catheter enabled careful monitoring of filling pressures and cardiac output. Non-invasive ventilation was no longer deemed necessary after the first forty eight hours. As the patient was hypotensive with a mean arterial pressure of $56 \mathrm{mmHg}$, and was noted to be volume-contracted with a mean right atrial pressure of $2 \mathrm{mmHg}$, cautious fluid boluses of $250-500 \mathrm{~mL}$ of crystalloid were given prior to doses of alpha-blockade. A test dose $(2.5 \mathrm{mg})$ of phentolamine was given with a cardiothoracic surgical team on standby, in case there was cardiovascular collapse and emergency mechanical circulatory support was required. Phentolamine was favoured over phenoxybenzamine as it is fast-acting, non-selective adrenergic antagonist and any adverse effects would be short-lasting compared to phenoxybenzamine, which has a halflife of approximately twenty four hours. No adverse 
cardiovascular effects were noted after the test dose of phentolamine and the patient was commenced on an initial dose of $2.5 \mathrm{mg}$ of phenoxybenzamine, followed by a dose of $5 \mathrm{mg}$ on the next day and $10 \mathrm{mg}$ once daily on the third day. After 6 days, the patient was stabilised on a dose of $20 \mathrm{mg}$ daily in divided doses $(0.4 \mathrm{mg} /$ $\mathrm{kg}$ ) prior to surgery and $2.5 \mathrm{mg}$ of bisoprolol due to tachycardia, which did not respond to additional fluid replacement, ruling out a reflex tachycardia. Prior to surgery on $20 \mathrm{mg}$ of phenoxybenzamine and $2.5 \mathrm{mg}$ of bisoprolol, the patient had a blood pressure which was persistently between 100/70 and $110 / 75 \mathrm{mmHg}$ with less than a $10 \mathrm{mmHg}$ drop in postural blood pressure and a heart rate of $<80 \mathrm{bpm}$ for two consecutive days. An elective laparoscopic left adrenalectomy was successfully performed on day 16 of admission. Intraoperatively, additional treatment with $7 \mathrm{mg}$ of metaraminol, a potent sympathomimetic amine, was used to manage hypotension. A further three litres of compound sodium lactate and $5 \mathrm{mg}$ of labetalol were also administered over the course of the surgery (Fig. 1D).

\section{Outcome and follow-up}

The patient made a complete recovery post-adrenalectomy and was discharged from the hospital on day 25. An echocardiogram prior to discharge revealed a normal ejection fraction of $65 \%$ and no regional wall motion abnormality. Plasma metanephrines were normalised (normetanephrine: $<270 \mathrm{pmol} / \mathrm{L}$, metanephrine: $<180 \mathrm{pmol} / \mathrm{L}$ ) in the post-operative period and the patient has scheduled endocrine and cardiology follow-up.

\section{Discussion}

This case demonstrates the importance of cross-disciplinary input in the complex management of a catecholamine crisis and clearly highlights the value of invasive monitoring to guide appropriate fluid resuscitation and alpha-blockade in this rare but challenging patient group. A recent review of the literature performed by Whitelaw et al. examined the use of alpha-blockade for the treatment of catecholamine crisis and found that mortality was significantly higher in those that did not receive treatment with alpha-blockade vs those that did (99\% vs 40\%) (4). Of course, the use of alpha-blockade may have been contraindicated in some of the reviewed cases because of co-existing hypotension resulting from cardiogenic shock and/or intravascular depletion. As outlined in the case we describe, careful monitoring of fluid status can guide appropriate fluid resuscitation and allow cautious introduction of alpha-blockade in a high dependency unit. We would also recommend that whenever possible, a patient should be monitored at a unit with expertise and access to mechanical circulatory support if needed. The use of intra-arterial balloon counter-pulsation or extra-corporeal life support has demonstrated efficacy in refractory cases of catecholamine crisis associated with hypotension (4) and can act as a bridging therapy to allow the catecholamine crisis to subside or provide the circulatory support needed to introduce alpha-blockade.

In this case, we have demonstrated that an initial test dose of a short-acting alpha-blocker, such as phentolamine, can provide valuable information about the cardiovascular reserve in these patients and that careful fluid resuscitation may be adequate to enable optimal alpha-blockade.

Close communication with experienced anaesthetic and surgical teams can allow for appropriate timing of elective surgery after medical optimisation, as this is now believed to result in superior outcomes when compared to emergency surgery $(3,4,9)$.

The case we describe provides a reminder of the precipitants that may exacerbate a catecholamine crisis. As illustrated herewith, our patient suffered a sudden deterioration following administration of intravenous contrast and intravenous glucocorticoids. It is suspected that the initial precipitant in this case was an adrenal haemorrhage as suggested by the haemorrhagic tumour on histology and the presenting symptom of acute abdominal pain.

Declaration of interest

The authors declare that there is no conflict of interest that could be perceived as prejudicing the impartiality of the research reported.

Funding

The authors thank the following funding bodies: Health Research Board Ireland (RC).

Patient consent

Written informed consent has been obtained from this patient and a copy has been submitted.

Author contribution statement

All authors contributed to the writing, editing and review of this manuscript. All authors were involved in the care and management of this patient. Dr Ruth Casey was responsible for creating Fig. 1 and Dr Stephen Pettit and Dr Catriona Bhagra were responsible for the creation of Fig. 2. 


\section{References}

1 Kopetschke R, Slisko M, Kilisli A, Tuschy U, Wallaschofski H, Fassnacht M, Ventz M, Beuschlein F, Reincke M, Reisch N, et al. 2009 Frequent incidental discovery of phaeochromocytoma: data from a German cohort of 201 phaeochromocytoma. European Journal of Endocrinology 161 355-361. (doi:10.1530/EJE-09-0384)

2 Newell KA, Prinz RA, Pickleman J, Braithwaite S, Brooks M, Karson TH \& Glisson S 1988 Pheochromocytoma multisystem crisis. A surgical emergency. Archives of Surgery 123 956-959.

3 Riester A, Weismann D, Quinkler M, Lichtenauer UD, Sommerey S, Halbritter R, Penning R, Spitzweg C, Schopohl J, Beuschlein F, et al. 2015 Life-threatening events in patients with pheochromocytoma. European Journal of Endocrinology 173 757-764. (doi:10.1530/ EJE-15-0483)

4 Whitelaw BC, Prague JK, Mustafa OG, Schulte KM, Hopkins PA, Gilbert JA, McGregor AM \& Aylwin SJ 2014 Phaeochromocytoma crisis. Clinical Endocrinology 80 13-22. (doi:10.1111/cen.12324)
5 Prejbisz A, Lenders JW, Eisenhofer G \& Januszewicz A 2011 Cardiovascular manifestations of phaeochromocytoma. Journal of Hypertension 29 2049-2060. (doi:10.1097/HJH.0b013e32834a4ce9) 6 Y-Hassan S 2016 Clinical features and outcome of pheochromocytoma-induced Takotsubo syndrome: analysis of 80 published cases. American Journal of Cardiology 117 1836-1844. (doi:10.1016/j.amjcard.2016.03.019)

7 Nef HM, Mollmann H, Akashi YJ \& Hamm CW 2010 Mechanisms of stress (takotsubo) cardiomyopathy. Nature Reviews Cardiology $\mathbf{7}$ 187-193. (doi:10.1038/nrcardio.2010.16)

8 Gagnon N, Mansour S, Bitton Y \& Bourdeau I 2017 Takotsubolike cardiomyopathy in a large cohort of patients with pheochromocytoma and paraganglioma. Endocrine Practice $\mathbf{2 3}$ 1178-1192. (doi:10.4158/EP171930)

9 Scholten A, Cisco RM, Vriens MR, Cohen JK, Mitmaker EJ, Liu C, Tyrrell JB, Shen WT \& Duh QY 2013 Pheochromocytoma crisis is not a surgical emergency. Journal of Clinical Endocrinology and Metabolism 98 581-591. (doi:10.1210/jc.2012-3020)

Received in final form 9 October 2017 Accepted 19 October 2017 